\title{
Comparison of UAV Image and UAV LiDAR for Construction of 3D Geospatial Information
}

\author{
Keun Wang Lee ${ }^{1}$ and Joon Kyu Park ${ }^{2 *}$ \\ ${ }^{1}$ Deptartment of Multimedia Science, Chungwoon University, 113, Sukgol-ro, Nam-gu, Incheon 22100, Korea \\ ${ }^{2}$ Deptartment of Civil Engineering, Seoil University, 28, Yongmasan-ro 90-gil, Jungnang-gu 02192, Seoul, Korea
}

(Received June 2, 2019; accepted August 9, 2019)

Keywords: accuracy, geospatial information, UAV LiDAR, UAV image, digital surface model

Geospatial information is data on the position of natural and artificial objects existing in space and is usually displayed in map form. Recently, virtual reality (VR) and augmented reality (AR) technologies have been utilized in various fields, and the importance of constructing three-dimensional spatial information as a basis of these technologies has increased. Light detection and ranging (LiDAR) technology is increasingly used to acquire data on terrain. Because LiDAR can generate a high-density point cloud while saving time and manpower consumption, it is increasingly utilized for 3D model building, measurement of structural deformation, and drawing construction for building maintenance. On the other hand, unmanned aerial vehicles (UAVs) are increasingly used for acquiring building geospatial information. In this study, geospatial information construction using UAV images and UAV LiDAR data was performed. Fixed-wing image acquisition and processing using UAV were performed. Data acquired using UAV LiDAR were used to construct a digital surface model (DSM), and the accuracy and characteristics of images and LiDAR data were analyzed. In the future, the construction of DSM using UAV LiDAR will greatly increase the efficiency of related field work.

\section{Introduction}

Geospatial information is an integral part of human life beyond the level of transmission of location information, and spatial information technology is evolving and developing into one that is tightly linked to life, thereby changing the lifestyle of individual human beings beyond the provision of various types of information. ${ }^{(1,2)}$ Recently, local changes in national territory and landscape have been occurring very frequently owing to rapid economic development, industrial structural changes, urban expansion due to development, and abnormal weather phenomena. ${ }^{(3,4)}$ These changes cause great problems in the efficient management of national territories and cities and in providing the latest spatial information required by industries and in certain social activities. ${ }^{(5,6)}$ Unmanned aerial vehicles (UAVs) can be used from time to time to acquire regular photos of various scales depending on flight altitude, as well as various types of image information such as oblique photographs and videos, which can be used very effectively. ${ }^{(7,8)}$

*Corresponding author: e-mail: jkpark@seoil.ac.kr https://doi.org/10.18494/SAM.2019.2466 
In particular, such data can be useful for construction sites. Construction sites are mostly distributed in urban areas as well as in mountains or farming and fishing villages where there is not much human or vehicle traffic. Several types of construction work must be carried out along various distances on the order of 10-100 m..$^{(9,10)}$ Surveys using existing GPS or total stations for measuring the status and quantity of construction sites involve so much time and manpower. ${ }^{(11)}$ In this study, 3D geospatial information of an effective terrain was planned and built using UAV images and UAV light detection and ranging (LiDAR) data. Figure 1 shows the study flow.

\section{Study Area and Data Acquisition}

In this study, a construction site in Incheon City was selected as the study area for the construction of 3D geospatial information using UAV images and UAV LiDAR data. The study area was found to be suitable for building geospatial information as it had a mixture of roads, land, and some forests. Data were acquired in three ways. Images were first acquired using fixed wings, and then data were acquired using UAV LiDAR. In addition, data were acquired using a 3D laser scanner to analyze the accuracy of the data acquired. Figure 2 shows the study area.

Data were acquired using fixed wings through Delair's UX11. The data were obtained at an altitude of $287 \mathrm{~m}$ for a total of $30 \mathrm{~min}$. The ground sample distance (GSD) was $4 \mathrm{~cm}$ and about 500 photos were acquired. Four ground control points (GCPs) were measured by the virtual reference station (VRS) method for georeferencing.

The UAV LiDAR sensor was used in the Surveyor Ultra of YellowScan, and data were acquired at an altitude of $60 \mathrm{~m}$ over a period of about $20 \mathrm{~min}$. UAV LiDAR is a 3D laser scanner for obtaining data with an inertial measurement unit (IMU) installed on the ground. Figures 3 and 4 show UX11 and SX10, respectively. ${ }^{(12)}$ Figure 5 shows the UAV LiDAR.

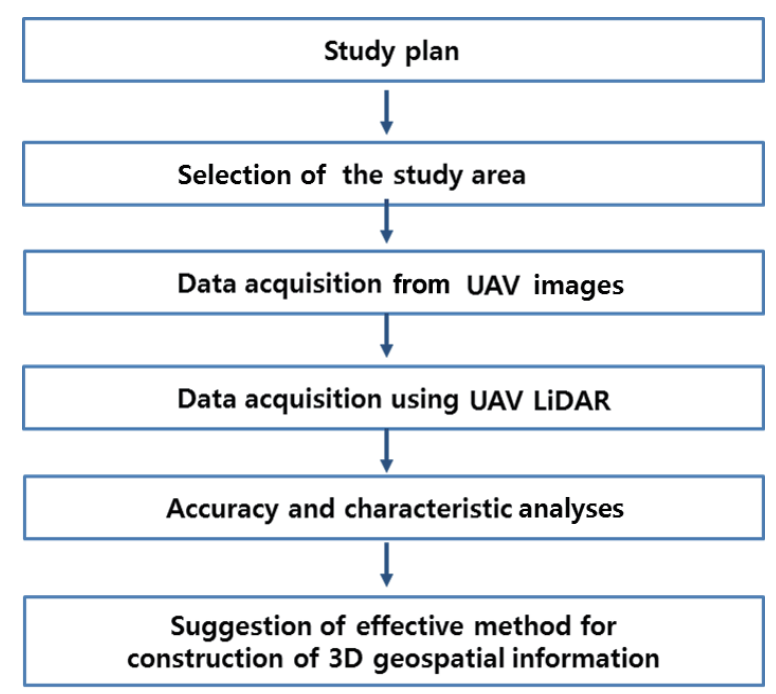

Fig. 1. Study flow. 


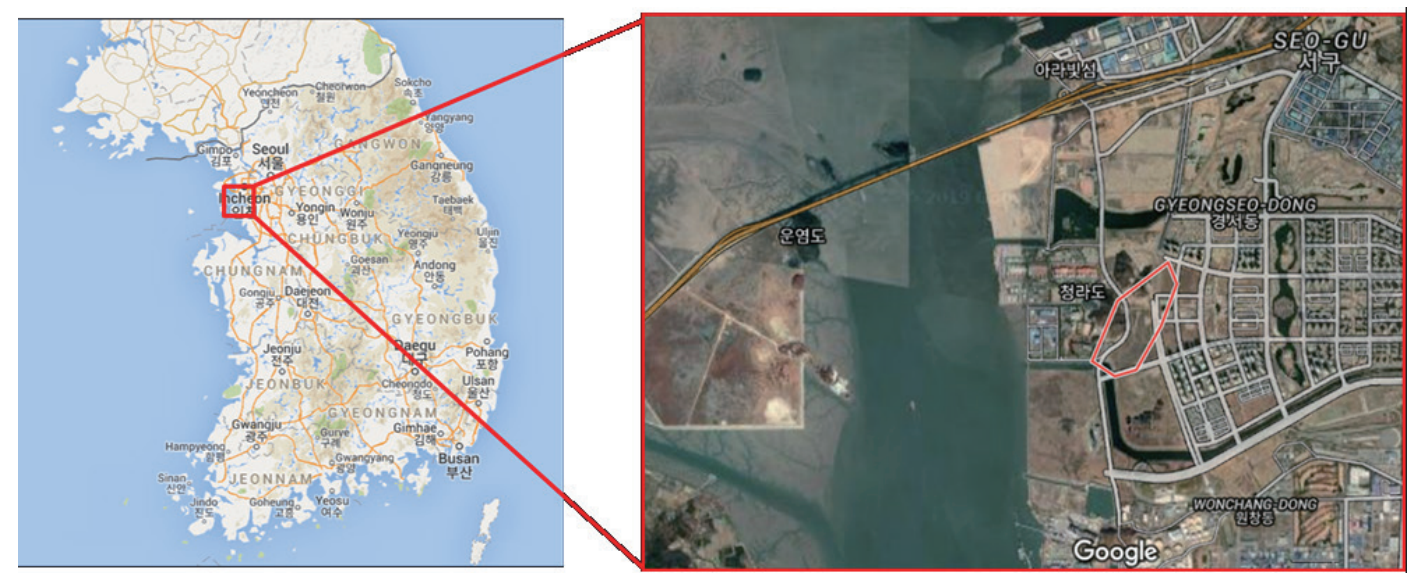

Fig. 2. (Color online) Study area.

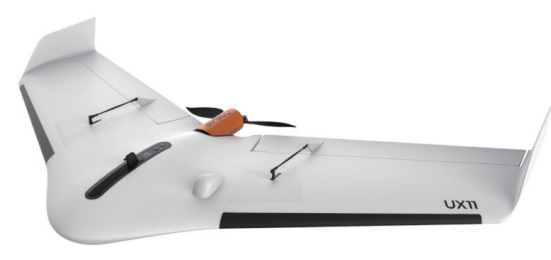

Fig. 3. (Color online) UX11.

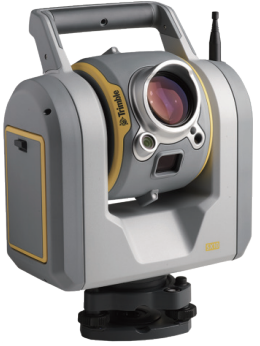

Fig. 4. (Color online) SX10.

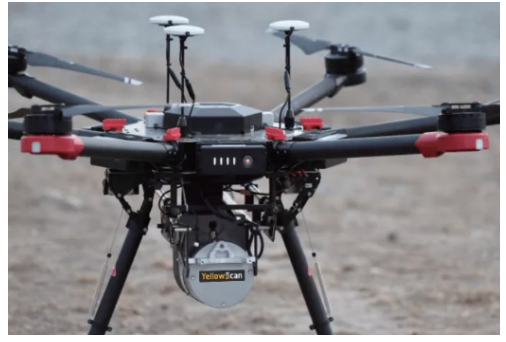

Fig. 5. (Color online) UAV LiDAR.

A 3D laser scanner was used to obtain reference data. Data were acquired using Trimble SX10 and used for the georeferencing and accuracy evaluation of the acquired the UAV images and UAV LiDAR data. Data were acquired for some of the study areas.

\section{Data Processing}

\subsection{UAV images}

The data acquired with UX11 included about 500 images and were processed using UAS Master Software. Data were processed by tie point extension, GCP measurement, orientation, and point cloud generation. No ortho images were generated because they were not used for comparison with UAV LiDAR, but the data processing results only generated a digital surface model (DSM) in the form of point clouds. Figure 6 shows the flow of UAV image data processing.

The DSM generated by data processing is shown in Fig. 7, which shows the data in the *las format in the form of a color point cloud, and 3D data of the study subjects such as roads, buildings, and forest were effectively generated. The 3D point cloud data acquired using SX10 were from some areas of the study site as reference data for accuracy assessment. Figure 8 shows the SX10 data. 


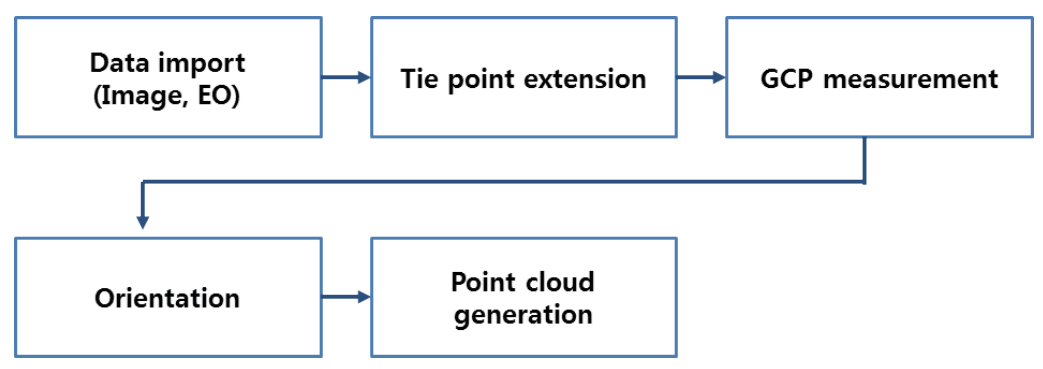

Fig. 6. Flow of UAV image data processing.

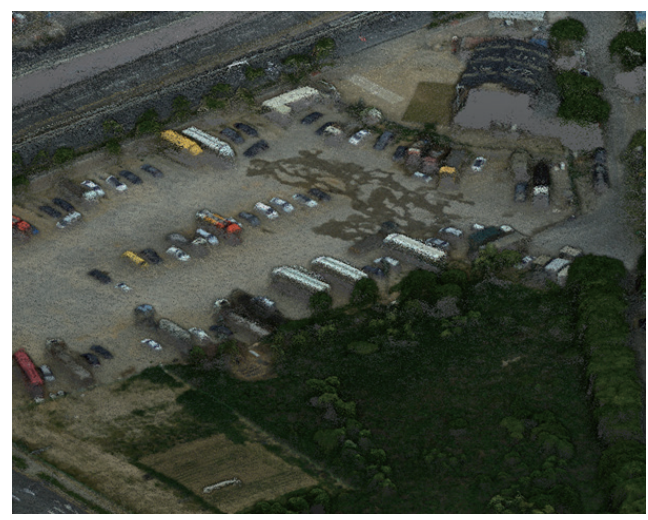

Fig. 7. (Color online) DSM generated using UAV images.

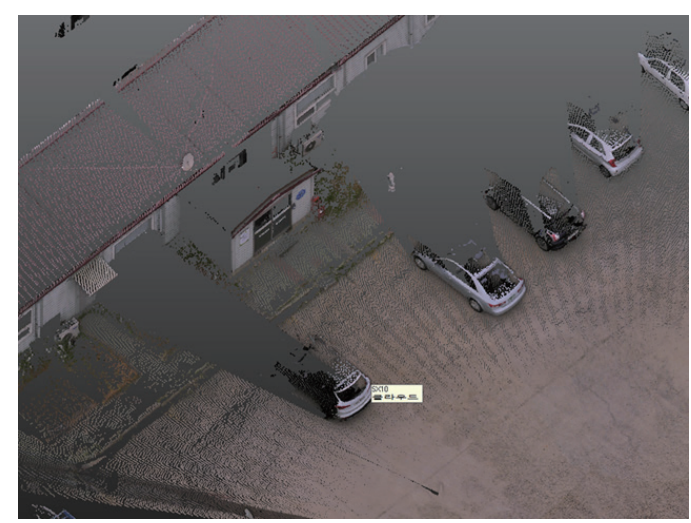

Fig. 8. (Color online) SX10 data.

\subsection{UAV LiDAR}

UAV LiDAR data were processed by preparing the flight path and creating the point cloud. Data processing of the flight path used the data of the base station and the GNSS and IMU data recorded in the UAV. After the processing of the flight path, the point cloud was generated using the UAV LiDAR data acquired along the flight path. Figure 9 shows the flow of UAV LiDAR data processing and Fig. 10 shows DSM generated using UAV LiDAR data.

\section{Analysis of Results}

\subsection{Accuracy analysis}

To evaluate the accuracy of DSM generated using UAV images, six check points were obtained from SX10 data, and the coordinates of the points were compared. Table 1 shows the results of the accuracy evaluation of the DSM generated using the UAV images.

The accuracy of the DSM generated using the UAV images showed a deviation of up to 8.4 $\mathrm{cm}$ from the DSM generated using the terrestrial LiDAR data. This deviation is small and the accuracy was sufficiently satisfactory for the 1:1000 digital map constructions. Therefore, the DSM generated using UAV images can be used in the production of digital maps.

In this study, SX10 data and shapes were compared to evaluate the accuracy of the UAV LiDAR data. Table 2 shows the results of the accuracy evaluation of the DSM generated using the UAV images, and Fig. 11 shows the overlap between UAV LiDAR data and SX10 data. 


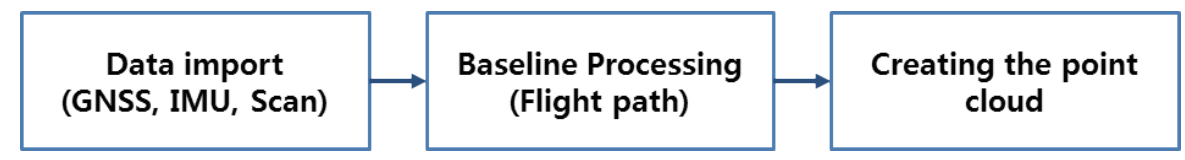

Fig. 9. Flow of LiDAR data processing.
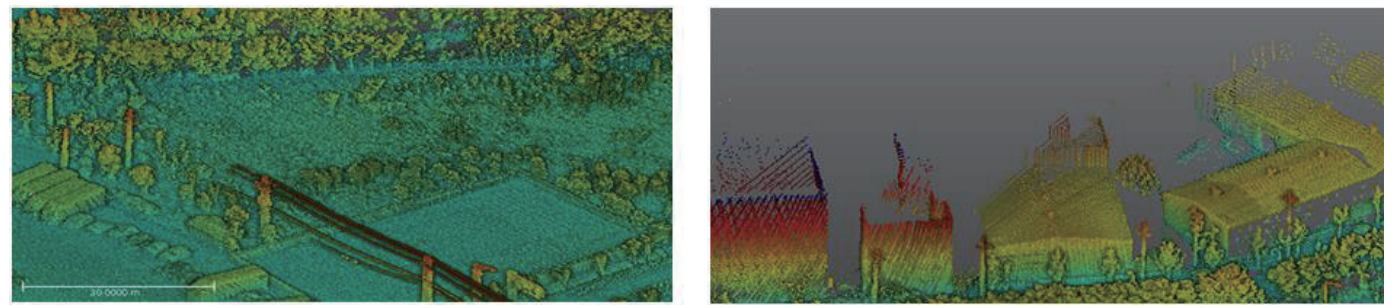

Fig. 10. (Color online) DSM generated using UAV LiDAR data.

Table 1

Accuracy analyses of UAV images.

\begin{tabular}{|c|c|c|c|c|c|c|c|c|c|}
\hline \multirow{2}{*}{$\mathrm{CP}$} & \multicolumn{3}{|c|}{ Reference } & \multicolumn{3}{|c|}{ UAV image } & \multicolumn{3}{|c|}{ Deviation } \\
\hline & $X(\mathrm{~m})$ & $Y(\mathrm{~m})$ & $H(\mathrm{~m})$ & $X(\mathrm{~m})$ & $Y(\mathrm{~m})$ & $H(\mathrm{~m})$ & $d X(\mathrm{~m})$ & $d Y(\mathrm{~m})$ & $d H(\mathrm{~m})$ \\
\hline 1 & 550964.494 & 167786.721 & 6.937 & 550964.517 & 167786.711 & 6.911 & -0.023 & 0.010 & 0.026 \\
\hline 2 & 550946.654 & 167767.081 & 10.111 & 550946.687 & 167767.101 & 10.188 & -0.033 & -0.020 & -0.077 \\
\hline 3 & 550876.096 & 16782.545 & 9.797 & 550876.084 & 16782.521 & 9.740 & 0.012 & 0.024 & 0.057 \\
\hline 4 & 551009.799 & 167796.097 & 19.703 & 551009.771 & 167796.076 & 19.787 & 0.028 & 0.021 & -0.084 \\
\hline 5 & 550872.045 & 167854.859 & 8.232 & 550872.044 & 167854.844 & 8.212 & 0.001 & 0.015 & 0.020 \\
\hline
\end{tabular}

Table 2

Accuracy analyses of UAV LiDAR.

\begin{tabular}{|c|c|c|c|c|c|c|c|c|c|}
\hline \multirow{2}{*}{$\mathrm{CP}$} & \multicolumn{3}{|c|}{ Reference } & \multicolumn{3}{|c|}{ UAV Image } & \multicolumn{3}{|c|}{ Deviation } \\
\hline & $X(\mathrm{~m})$ & $Y(\mathrm{~m})$ & $H(\mathrm{~m})$ & $X(\mathrm{~m})$ & $Y(\mathrm{~m})$ & $H(\mathrm{~m})$ & $d X(\mathrm{~m})$ & $d Y(\mathrm{~m})$ & $d H(\mathrm{~m})$ \\
\hline 1 & 550964.494 & 167786.721 & 6.937 & 550964.513 & 167786.742 & 6.918 & -0.019 & -0.021 & 0.019 \\
\hline 2 & 550946.654 & 167767.081 & 10.111 & 550946.676 & 167767.112 & 10.155 & -0.022 & -0.031 & -0.044 \\
\hline 3 & 550876.096 & 16782.545 & 9.797 & 550876.091 & 16782.531 & 9.788 & 0.005 & 0.014 & 0.009 \\
\hline 4 & 551009.799 & 167796.097 & 19.703 & 551009.783 & 167796.066 & 19.737 & 0.016 & 0.031 & -0.034 \\
\hline 5 & 550872.045 & 167854.859 & 8.232 & 550872.049 & 167854.831 & 8.224 & -0.004 & 0.028 & 0.008 \\
\hline
\end{tabular}

The green dots in Fig. 8 represent the SX10 data, and the yellow dot represents the UAV LiDAR data. The accuracy of the UAV LiDAR data was approximately $5 \mathrm{~cm}$ compared with that of the SX10 data. This is considered to be due to the noise characteristics of the sensor, and filtering will improve accuracy.

\subsection{Comparison of characteristics of DSMs}

In this study, DSMs were generated using UAV images and UAV LiDAR data. For DSM generated using UAV images, it took approximately $30 \mathrm{~min}$ to acquire data from the study site and data were acquired in a wider area than in the case UAV LiDAR. However, as seen in Fig. 12, there were features where no data were generated for the region where no images were acquired. Figure 12 shows DSMs of the same region generated using UAV images and LiDAR data. 


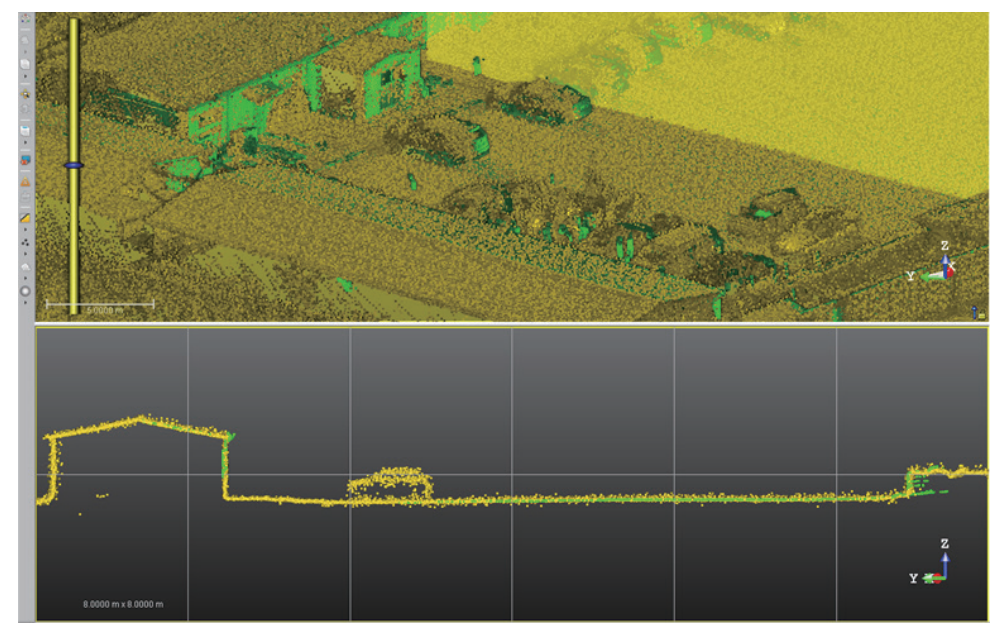

Fig. 11. (Color online) Overlap between UAV LiDAR data and SX10 data.

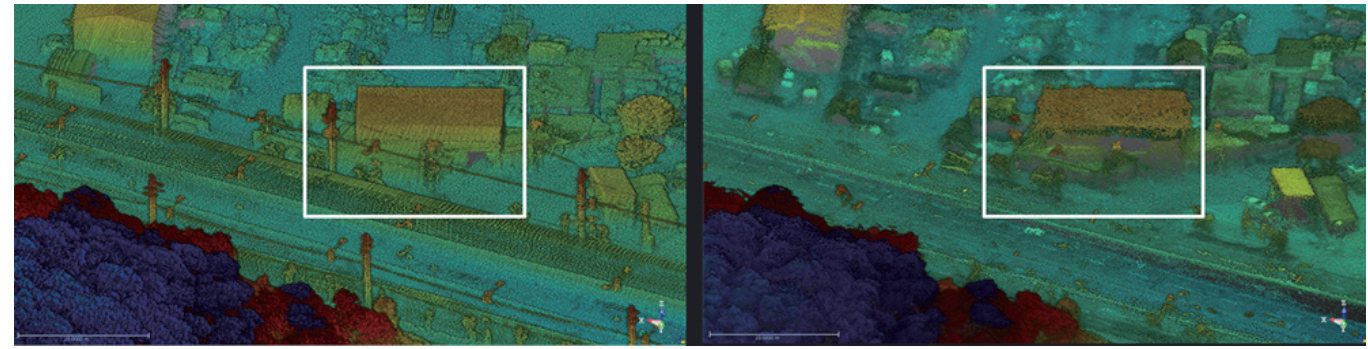

(a)

(b)

Fig. 12. (Color online) DSMs of the same region generated using (a) UAV LiDAR data and (b) UAV images.

As shown in the white area in Fig. 12, the UAV image did not generate data on shaded areas where the images were acquired during the processing of the data. These results indicate that weather-induced shadows can also affect acquisition data. The method using UAV LiDAR can acquire more data than that using UAV images because the data are acquired by 3D laser scanners. UAV LiDAR will also be more useful because data on trees and ground are acquired together in forest areas. Figure 13 shows forest areas acquired with UAV LiDAR and the ground extraction result.

\subsection{Efficiency analysis of workflow}

Data acquired with UAV LiDAR in forest areas have both tree and ground data, so data of only the ground can be extracted by removing data of trees. The extracted ground is a digital element model (DEM), which is the most important data utilized in construction work. As shown in Fig. 10, the ground on which the trees have been removed can be observed in detail, such as the creases of the ground. More detailed data can be obtained using UAV LiDAR than by conventional GPS methods, and the areas obscured by trees can be observed. Table 3 shows data acquisition and processing time and workflow for methods using UAV images and UAV LiDAR data. 


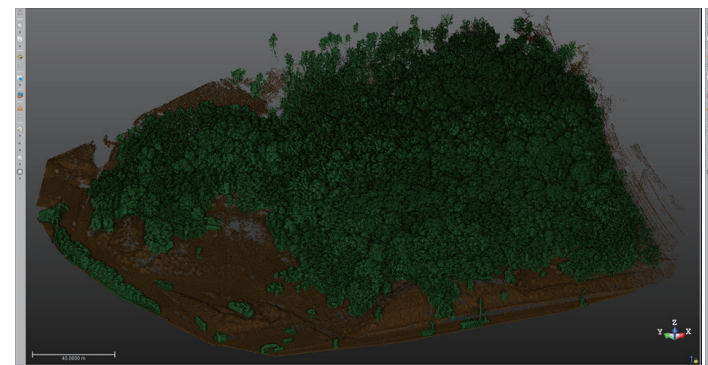

(a)

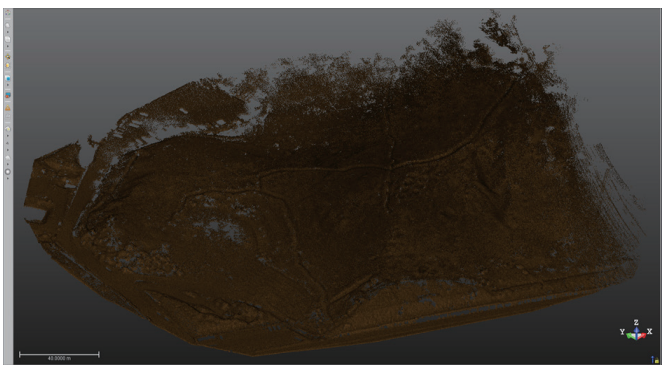

(b)

Fig. 13. (Color online) Forest areas acquired with (a) UAV LiDAR data and (b) ground extraction result.

Table 3

Data acquisition and processing time and workflow.

\begin{tabular}{lcc}
\hline Workflow & UAV Image (h) & UAV LiDAR (h) \\
\hline GCP survey & 1.0 & 0.1 \\
Data acquisition & 0.5 & 0.8 \\
Tie point extraction & 2.0 & - \\
Orientation & 0.5 & - \\
Postprocessing of flight path & - & 0.1 \\
Generation of point cloud & 2.0 & 0.9 \\
Total & 6.0 & 1.9 \\
\hline
\end{tabular}

The data processing time for the same area is about three times faster by the UAV LiDAR method than by using UAV images. These results are those for a study area of $0.25 \mathrm{~km}^{2}$, and it is expected that the UAV LiDAR's work efficiency will increase as the area of the target site increases. Methods using UAV images and UAV LiDAR data have their respective characteristics and advantages. The method using UAV images enabled data acquisition over a wide area in a short time, and it was possible to generate color point cloud data using the images. The method using UAV LiDAR data took more time to acquire data than that using UAV images, but data processing can be completed more quickly. It is also expected to be more useful in construction work as data on shaded areas can be obtained from the images.

\section{Conclusions}

In this study, geospatial information construction using UAV images and UAV LiDAR data is performed. By using UAV images, we effectively generated the DSM of the study target. The results also suggest that this method can be used in the production of digital maps by verifying that the DSM generated using UAV images has an accuracy of about $8 \mathrm{~cm}$. The accuracy of the DSM generated using UAV LiDAR data was approximately $5 \mathrm{~cm}$ compared with the reference data. This is considered to be due to the noise characteristics of the sensor, and filtering will improve accuracy. The DSM was generated using UAV LiDAR data, and each characteristic was analyzed in comparison with that of the DSM generated using UAV images. The use of UAV images did not generate data on shaded areas if the images were not acquired during the processing of the data. However, UAV LiDAR can provide more data than the UAV because the data are acquired by 3D laser scanners. UAV LiDAR generated data about 3 times faster 
than UAV. The larger target area, the greater the difference in efficiency. The method using UAV LiDAR data will be highly efficient at construction sites because there is no shaded area compared with the method using UAV images, and the generation of DEM through "deforestation" is possible. In the future, the construction of DSM using UAV LiDAR will greatly increase the efficiency of related field work.

\section{Acknowledgments}

This research was supported by the Basic Science Research Program through the National Research Foundation of Korea (NRF) funded by the Ministry of Science and ICT (No. NRF2018R1C1B6004021).

\section{References}

1 D. G. Lee and D. C. Lee: J. Korean Soc. Surv. Geod. Photogramm. Cartography 36 (2018) 355. https://doi. org/10.7848/ksgpc.2018.36.5.355

2 J. H. Seong, Y. K. Han, and W. H. Lee: J. Korean Soc. Surv. Geod. Photogramm. Cartography 36 (2018) 279. https://doi.org/10.7848/ksgpc.2018.36.4.279

3 H. Woo, S. Beak, W. Hong, M. Chung, H. Kim, and J. Hwang: Spatial Inf. Res. 26 (2018) 639. https://doi. org/10.1007/s41324-018-0208-9

4 O. S. Song and J. R. Koo: Trans. Korean Soc. Noise Vib. Eng. 28 (2018) 181. https://doi.org/10.5050/ KSNVE.2018.28.2.181

5 J. H. Oh, Y. J. Jang, and C. N. Lee: J. Korean Soc. Surv. Geod. Photogramm. Cartography 36 (2018) 565. https://doi.org/10.7848/ksgpc.2018.36.6.565

6 Z. Hu, S. Wei, and J. Jiang: J. Phys. 910 (2017) 1. https://10.1088/1742-6596/910/1/012046

7 K. B. Kang, H. S. Kim, J. W. Jwa, H. C. Kim, and M. J. Kang: J. Korean Academia-Industrial Cooperation Soc. 18 (2017) 117. https://doi.org/10.5762/KAIS.2017.18.10.117

8 J. K. Park and K. Y. Jung: J. Korean Academia-Industrial Cooperation Soc. 19 (2018) 602. https://doi. org/10.5762/KAIS.2018.19.2.602

9 J. S. Um: Spatial Inf. Res. 26 (2018) 143. https://doi.org/10.1007/s41324-018-0163-5

10 K. R. Lee, J. H. Seong, Y. K. Han, and W. H. Lee: J. Korean Soc. Surv. Geod. Photogramm. Cartography 37 (2019) 9. https://doi.org/10.7848/ksgpc.2019.37.1.9

11 G. H. Kim, S. W. Park, M. J. Kim, D. S. Lee, and H. S. Choi: J. Korean Tunnelling and Underground Space Assoc. 19 (2018) 121. https://doi.org/10.9711/KTAJ.2017.19.2.121

12 Trimble Inc.: http:www.trimble.com (accessed April 2019).

\section{About the Authors}

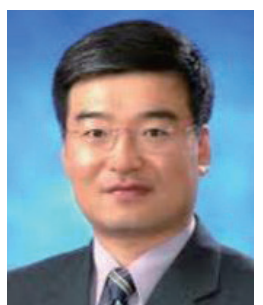

Keun Wang Lee is a professor of the Deptartment of Multimedia Science at Chungwoon University. He received his B.S. degree in electronic computing from Hanbat University in 1993 and his M.S. and Ph.D. degrees in computer science from Soongsil University, Korea in 1996 and 2000, respectively. His research interests are in the areas of multimedia communication and multimedia application. (kwlee@chungwoon.ac.kr)

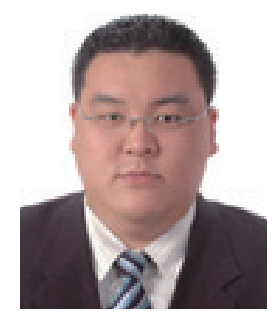

Joon Kyu Park is currently a professor of the Deptartment of Civil Engineering at Seoil University. He received his B.S., M.S., and Ph.D. degrees in civil engineering from Chungnam National University, Korea in 2001, 2003, and 2008, respectively. His research interests are in the areas of GPS and geo-spatial information engineering. (jkpark@seoil.ac.kr) 